\title{
L'Italia che non si vuole conoscere
}

\author{
Anna Zembrino \\ Universitat de Barcelona \\ annazembrino@ub.edu
}

"L'Italia è multilingue e non lo sa, e nemmeno vuol troppo saperlo». Così T. De Mauro, nella sua Prefazione al recente volume di Anna Bogaro, ${ }^{1}$ riflettendo sulla comune cattiva coscienza italiana che, come ha dato a lungo per scontato che letteratura italiana sia quella che si esprime in lingua italiana, così ha dato tardi attuazione all'articolo 6 della nostra Costituzione (anno 1948: «la Repubblica salvaguarda con apposite norme le minoranze linguistiche»): solo nel 1999 con la legge 482 a tutela delle minoranze linguistiche storiche, che interessano il 5\% della cittadinanza italiana. A illustrare la storia delle testimonianze e la consistenza attuale della produzione letteraria in alcune di queste lingue di minore diffusione storicamente presenti nel territorio della penisola (da secoli: come l'albanese, la greca, la slovena, o da sempre: come la sarda, l'occitana, la ladina, la friulana), è dedicato il saggio di A. Bogaro, che per la prima volta offre un quadro d'insieme su questo mondo letterario plurilingue attivo entro i nostri confini e solitamente sconosciuto al grande pubblico e non molto frequentato neppure dalla critica.

Il volume si apre con un capitolo 'metodologico' dedicato alla questione lingua/dialetto nella letteratura italiana nella prospettiva della critica novecentesca. Per l'autrice, sullo sfondo opera la distinzione di B. Croce tra letteratura dialettale spontanea e letteratura dialettale d'arte o riflessa e la sua considerazione che le letterature dialettali sarebbero potute divenire nazionali, se ci fossero stati centri culturali autonomi come Portogallo e Catalogna nella penisola iberica, ma così non è stato e il critico interpreta come contributo all'unificazione, nel Seicento come dopo l'Unità, la reciproca scoperta delle varie regioni d'Italia attraverso la loro produzione letteraria dialettale d'arte, che non mette in discussione la supremazia dell'italiano. Un'idea di non antagonismo ma «fecondo dialogo con i modelli elaborati dagli autori in lingua» (p. 19), che anche un valente studioso di letteratura dialettale quale è F. Brevini fa sua: in poesia,

1. Anna Bogaro, Letterature nascoste. Storia della scrittura e degli autori in lingua minoritaria in Italia, Roma: Carocci, 2010, p. 12. 
produzione in lingua e in dialetto sono «le due facce di una medesima tradizione letteraria» (Ibid.).

È noto che nella storia politicamente divisa del nostro paese la letteratura ha rappresentato il più forte elemento unitario. Nel secondo dopoguerra però, venuti meno i forzosi argini ideologici del fascismo, i rapporti reciproci di lingua e dialetto nel passato come nel presente, nel parlato come nella scrittura letteraria, o le ragioni della perennemente risorgente 'questione della lingua', così tipicamente nostrana, sono stati indagati e ormai chiaramente delineati da studi fondamentali che hanno dimostrato, in primis quelli di C. Dionisotti, l'inevitabilità dell'inserimento di una categoria fondamentale nello studio della storia della letteratura italiana: la 'geografia', istanza recepita nell'impianto della Letteratura italiana diretta da Asor Rosa e pubblicata da Einaudi tra il 1982 e il 2000. Ma nonostante questa articolazione 'polifonica' della nostra storia letteraria inizi finalmente ad essere accolta a livello più generale, l'autrice rileva nondimeno che «le tradizioni letterarie prodotte nelle lingue minoritarie non trovano udienza in quanto tali nelle trattazioni generali» (p. 17-18). Una prima difficoltà viene indicata dall'autrice nella definizione stessa di 'letteratura minore', in quanto nella bibliografia critica, — ampiamente passata in rassegna nella prima sezione del saggio per ricostruire lo stato dell'arte manca completamente ogni riferimento al concetto di 'lingua minoritaria': «Nel calderone sempre bollente della questione lingua/dialetto le lingue 'altre' storicamente presenti nella penisola da secoli, confluiscono nel mare magnum dialettale» (p. 52) con rare eccezioni. Sociolinguisticamente, la stessa condizione di 'minoranza' va meglio precisata: sempre intesa rispetto a una maggioranza, per T. Telmon essa va stabilita caso per caso (ad es. nelle isole linguistiche tedesche in Friuli, maggioritario è il friulano rispetto all'enclave germanofona), tenendo anche conto della eventuale presenza di una koiné o lingua standard di riferimento, mentre G. Francescato preferisce definire minoranza un gruppo (per solito non molto numeroso) di alloglotti che nella prima socializzazione acquisisce come lingua madre una lingua diversa da quella nazionale (p. 54) e su questa definizione ci pare si attesti l'autrice. La minoranza può essere omogenea e compatta, come quella friulana, o diffusa, come quella albanese in Sicilia e Calabria, e un ulteriore elemento di diversificazione è la presenza o meno di una lingua-tetto che sia lingua di Stato, come nel caso dello sloveno. Questo in un'ottica linguistica. Ma cosa succede quando una minoranza produce letteratura? È letteratura minore quella prodotta in una lingua minore? Non per G. Deleuze e F. Guattari che vedono l'emblema della letteratura minore nel tedesco di Praga usato dall'ebreo Franz Kafka e individuano uno dei suoi caratteri principali nel fatto che in essa tutto è politica, perché quello che l'autore dice ha suo malgrado una funzione collettiva ed è "necessariamente politico, anche se gli altri non sono d'accordo» (p. 56). Da questo loro teorema deriva un doppio corollario: lo scrittore di letteratura minoritaria ha la possibilità di emergere individualmente anche se non particolarmente dotato di talento - corollario che l'autrice segnala valere per tutte le letterature di cui in seguito tratta - e ha già in partenza la 
possibilità di costruire una comunità virtuale e potenziale; la letteratura minore è per Deleuze e F. Guattari una possibilità rivoluzionaria che cresce in seno a quella maggiore, a patto che lo scrittore trovi «il proprio punto di sottosviluppo, un proprio dialetto, un terzo mondo, un deserto tutto per sé» (Ibid.). Sulla visibilità o meno delle lingue e letterature minori pesano ovviamente fattori extralinguistici, a proposito del sardo, ad es., G. Carongiu li individua nell'ideologia della classe dirigente isolana e negli opinion makers culturali urbani-metropolitani che la esprimono, i quali «azzerano il prestigio sociale della limba (lingua) e delle sue espressioni, comprese naturalmente quelle letterarie», (p. 58) ma per fortuna spazi di valorizzazione per i lesser used languages e relativi patrimoni culturali si trovano nella comune casa europea: il dato identitario, che può suscitare polemiche, attriti, diffidenze in ambito regionale/nazionale, in Europa diviene semplicemente uno dei tanti tasselli di diversità che formano la fisionomia del continente, che vanno preservati e valorizzati, ed è questo forse il migliore orizzonte di riferimento anche per lo scrittore italiano che sceglie di usare una lingua minore (Sergio Atzeni dichiarava di sentirsi all'unisono sardo, italiano, europeo) (p. 87), senza dimenticare poi che uno degli aspetti che rende particolarmente interessante occuparsi di questo argomento è la sua contiguità con la letteratura di migrazione, che pone analoghi problemi di metodo, e la letteratura translingue in genere, una letteratura che «scuote le fondamenta degli antichi palazzi delle tradizioni letterarie europee ... e dai sottoscala di tali palazzi fanno capolino altre letterature che parlano nelle lingue minoritarie d'Europa» (p. 69) che riconosciute o meno giuridicamente, sempre si trovano in posizione sociologicamente subalterna, ma possono suggerire contatti e prospettive inedite e feconde.

Il volume, dopo il primo ampio capitolo, traccia una rapida storia e il panorama attuale per le lingue minori che hanno prodotto una letteratura scritta: sardo, ladino dolomitico, sloveno, friulano, occitano, arbëreshe e si chiude con sei interviste ad altrettanti autori rappresentativi di ciascuna di esse (Flavio Soriga, Roland Verra, Boris Pahor, Carlo Tolazzi, Franco Bronzat, Carmine Abate). Va subito detto che non in tutte si riscontra la stessa vivacità e consistenza, il che dipende certo dalla diversa consistenza numerica delle rispettive minoranze, ma anche dai loro diversi percorsi di 'autocoscienza' e dalle peculiari vicende storiche. Nel caso della letteratura occitana delle valli alpine del Piemonte occidentale, per esempio, se le prime attestazioni risalgono al 1226-28 (quindi all'altezza cronologica del Cantico delle creature di San Francesco), tra il XIV e XVI secolo l'influsso valdese, di cui risentono, attirerà su di loro l'attenzione della censura cattolica e i testi religiosi non saranno più composti in lingua d'oc a partire dal Cinquecento, quando il francese diventerà lingua ufficiale della zona anche in ambito ecclesiastico e i segni di un vero risveglio letterario si avranno molto tardi, appena negli anni Sessanta del Novecento quando, insieme a una nuova fioritura poetica, si fa strada nei valligiani la consapevolezza che il loro "parlare a modo nostro" non meglio specificato (p. 170), è una lingua millenaria, quella che ha dato all'Europa tutta le forme e i temi del suo immaginario poetico romanzo. Se nel caso occitano vediamo 
la Chiesa esercitare una influenza inibitrice, in altri invece gli ecclesiastici hanno avuto un ruolo fondamentale nella valorizzazione della lingua minore e del suo patrimonio culturale. È per esempio il caso dell'arbëreshe, la minoranza albanese che si insedia nel sud d'Italia a partire dal sec. Xv, in contemporanea con l'invasione ottomana (e la successiva caduta di Scutari nel 1479), al tempo del re di Napoli Alfonso I d'Aragona, e poi del figlio e suo successore Ferdinando I, che ne favorì l'insediamento per l'aiuto ricevuto contro il rivale Giovanni d'Angiò dai condottieri albanesi, tra i quali il mito nazionale albanese: Giorgio Castriota Scanderbeg. Gli albanesi pur cattolici erano però di rito bizantino in lingua greca e questo causò a volte frizioni con la Chiesa latina, ma nel sec. XVIII la salita al pontificato di Clemente XI (1700-1721), con rivendicate origini albanesi per parte di padre, significò un momento propizio alla conservazione e valorizzazione dell'albanese, con la successiva fondazione del Collegio Corsini a San Benedetto Ullano (nel 1732, poi trasferito a San Demetrio Corone presso il Collegio S. Adriano nel 1794) e a Palermo del Seminario greco-albanese (nel 1735), istituzioni nelle quali si formarono «intellettuali e clerici progressisti, che svolsero un ruolo di protagonisti nel movimento risorgimentale italiano» (p. 174), tra i quali Francesco Crispi, albanese di Sicilia, garibaldino e futuro Presidente del Consiglio dei Ministri (p. 177). Il plurilinguismo è la cifra che accomuna la situazione della Sardegna e della Ladinia dolomitica. Dal momento che in Italia, tradizionalmente, nelle storie letterarie non entra la letteratura orale (di cui si occupano gli studiosi di etnografia e tradizioni popolari), le leggende e la poesia epicocavalleresca ladine, autoctone e antiche, non hanno diritto di cittadinanza e non possono fornire certificati di nascita letteraria (e lo stesso discorso vale per le villotte friulane), che viene posta pertanto dagli studiosi tra fine Ottocento e inizi Novecento, mentre per una "dignità letteraria conclamata» alcuni preferiscono il secondo dopoguerra (p. 98). Nemmeno i ciantastòries nel loro continuo vagare di valle in valle e rimescolare varianti linguistiche, riuscirono a dar vita ad una koinè delle sei valli dolomitiche, a lungo divise dai confini tracciati dalla politica, e il policentrismo è rimasto un tratto della letteratura ladina dolomitica (nelle sue varianti gardenese, badiotto/marebbano, fassano e fodom, più il ladin dolomitan, la lingua ladina scritta unificata) insieme al trilinguismo dei valligiani in generale e degli scrittori in particolare, che spesso optano anche per il tedesco o l'italiano, con scelte consapevoli, nelle quali al ladino è spesso demandato il compito di rintracciare le radici perdute (p. 62). Anche in Sardegna dicevamo, come in Ladinia, la parola aggregante è plurilinguismo. Nell'isola la lingua sarda, dalle caratteristiche così specifiche, ebbe un' iniziale processo espansivo, dato il suo uso ufficiale fin dai primordi accanto al latino nei documenti dei giudicati di Torres, Gallura, Arborea e Cagliari in cui era divisa nel sec. xI la Sardegna postbizantina. Anche quando nei secoli XIII e XIV l'isola è percorsa da trovatori provenzali e italiani, tacciono i documenti letterari e la lingua sarda sembra conservare caratteristiche pratico-legislative. Ma con la caduta dell'ultimo giudicato (guerra di Arborea 13551410) e l'arrivo degli aragonesi, inizia il periodo propriamente feudale segna- 
to da un gravissimo decremento demografico dovuto a ripetute carestie ed epidemie. Troviamo qui, nella precarietà economico-sociale che ne deriva e nei forti condizionamenti esterni, le ragioni che minano essenzialmente le condizioni per lo sviluppo successivo della lingua sarda e ne causano la subalternità alle lingue nazionali venute dall'esterno. Nel Cinquecento troviamo Cagliari catalanizzata in ambito giuridico, ma sarda a livello popolare, con il castigliano in espansione; a Sassari una situazione conflittuale tra sardo logudorese, catalano e castigliano, con l'italiano minoritario ovunque e la Corona spagnola che preme per l'uso pubblico del castigliano, in questo agevolata dal fatto che i riferimenti gerarchici della Chiesa cattolica vengono spostati dalla penisola italica a quella iberica. La Corona ottiene il successo della sua politica linguistica nel corso del Seicento che vede un'ampia ispanizzazione, anche se il sardo si conserva a livello popolare mantenendo un unico ambito pubblico: quello religioso (p. 75). Nel 1720, dopo una breve parentesi austriaca, l'isola passa ai Savoia e resta ispanizzante fino al 1760, quando l'uso pubblico del castigliano viene proibito, i maestri ispanofoni sostituiti con italofoni (gesuiti e scolopi) e inizia così un'italianizzazione perlomeno contraddittoria con quella che è la realtà linguistica nel cuore del regno, in Piemonte, e nella Corte stessa, dove non è l'italiano ma il francese la lingua usata (chi non ricorda le fatiche dell'Alfieri, antesignano della risciacquatura di panni in Arno, nella sua eroica conquista dell'idioma gentile?). Ma la limitazione sabauda del castigliano sembra giovare al sardo che ricompare nella poesia scritta (in quella orale non era mai venuto meno) e l'entrata nel Regno d'Italia porterà un miglioramento nel settore dell' istruzione e nella diffusione della stampa. Nella narrativa sarda tra Otto e Novecento abbondano i romanzi storici, ma questo è tratto comune con le altre letterature minori trattate nel volume e ben si spiega con il bisogno di far conoscere vicende e personaggi della propria storia ad un pubblico il più possibile ampio. Non tentiamo neppure di riassumere l'abbondanza di dati e riferimenti presentati al lettore riguardo alla produzione in prosa del secondo dopoguerra per tutte le letterature minori trattate e in particolare per la letteratura sarda, forse la più ricca da questo punto di vista. Passando ad altra area, viene spontaneo accostare alla Sardegna il Friuli. Intanto per le peculiarità della lingua, in questo caso determinate non dall'isolamento geografico ma dalla separazione politica: il Friuli è governato tra il 1077 e il 1251 da patriarchi ghibellini di origine (e quindi di lingua) tedesca, e forse proprio nella mancata possibilità da parte della popolazione (di lingua romanza) di un 'controllo' del proprio eloquio misurato sulla parlata della classe dominante è da vedere una delle ragioni delle vistose peculiarità del friulano rispetto agli altri esiti italoromanzi, come ipotizzato da G. Francescato. È il friulano lingua adatta alla letteratura? No. Questa la perentoria condanna di Dante nel De vulgari eloquentia che stigmatizzando la dura pronuncia del famoso Ces fas-tu di aquileiesi e istriani, al contempo ne attesta un uso sovra regionale. Sappiamo che le carte friulane erano numerose nel Trecento e i primi testi poetici sono della metà dello stesso secolo. Ma con l'avvento del dominio della Repubblica di Venezia (1420) il Friuli si trova diviso tra l'influenza della 
Serenissima nella sua parte occidentale e quella asburgica nella sua parte orientale, con una divisione destinata a durare. In questo nuovo quadro il friulano «tende a sparire quasi del tutto dagli usi scritti pratici e trova udienza nella cornice della polemica letteraria antitoscana, assecondando un sentimento comune ad altre realtà peninsulari» (p. 136). I versi in friulano vogliono esprimere un'identità 'altra', ma si muovono nel solco della letteratura dialettale riflessa, nonostante le rivendicazioni, senza indicare un riscatto socio-linguistico del friulano, che anzi continua nel corso del Cinquecento a regredire nell'uso cittadino e comune come segnala R. Pellegrini (p. 137). Il Seicento è il secolo della 'meraviglia' e degli esercizi accademici, dominato dalla figura di Ermes di Colloredo (1622-1692); l'Ottocento, anch'esso ricco di produzione letteraria, dà uno strumento prezioso: il Vocabolario friulano di J. Pirona, uscito postumo nel 1871 (e poi più volte aggiornato) e la cifra dell'anima friulana sembra essere concordemente rappresentata da P. Zorutti con il suo elogio dell'antico ordine sociale, di un mondo contadino e piccolo borghese sostanzialmente immobili e conservatori. Ma diversa è la temperie che si respira nel Friuli orientale. Udine e Gorizia sono sempre state i due poli dell'anima friulana, ma con comportamenti molto diversi rispetto alla lingua friulana: se Udine, sotto dominio veneziano, è venuta sviluppando un ben noto senso d'inferiorità, Gorizia, sotto il dominio dell'impero austro-ungarico, questo senso d'inferiorità non lo ebbe mai e come osserva G. Faggin «il friulano fu tenuto in onore a tutti i livelli e non fu visto come uno scomodo concorrente dell'italiano, ma semmai come un suo dialettico complemento» (p. 144). Per avere un'idea della vivacità intellettuale che si poteva respirare in quelle terre basta pensare a quel monumento della dialettologia italiana che sono i Saggi ladini di G. I. Ascoli, ebreo goriziano, illustre studioso fondatore dell'Archivio Glottologico Italiano il cui primo numero, nel 1873, contiene il celebre Proemio, con le più lungimiranti osservazioni a proposito della rinata ottocentesca questione della lingua. Questo fu vero fino all'annessione del Friuli orientale al Regno d'Italia nel 1918; prima i migliori intellettuali goriziani uscivano tutti dal K. K. Staatsgymnasium (Imperial Regio Ginnasio Statale), dove al mattino la lingua veicolare era il tedesco, ma al pomeriggio «gli studenti italiani/friulani e sloveni potevano seguire dei corsi facoltativi nelle rispettive lingue» (Ibid.). La cultura tedesca era alla base della loro formazione e il bilinguismo era un'ottima ginnastica mentale che stimolava il senso critico, così, - è sempre G. Faggin che parla —, "gli aspetti deteriori della cultura italiana (la tabe retorica, la superficialità, la mancanza di impegno sociale) venivano neutralizzati» (Ibid.). Molte altre pagine interessanti del saggio raccontano la ricchezza di esperienze culturali del goriziano e delineano, ormai in pieno secondo conflitto mondiale, la svolta causata dall'impatto della presenza di P. P. Pasolini a partire dal 1942 a Casarsa, e tutta la successiva produzione in friulano, che da quella esperienza non può prescindere e con essa deve confrontarsi; ma vorremmo brevemente far cenno all'ultima delle letterature trattate nel libro di A. Bogaro, quella slovena, le cui dinamiche e la cui storia divergono da quelle finora esaminate e più delle altre dovrebbero forse farci riflettere 
sulla nostra comune cattiva coscienza. La letteratura slovena del cosiddetto Litorale (Gorizia, Trieste e i loro territori, cui si può aggiungere la Slavia friulana) ha una storia che rispecchia i rapporti complessi che in quelle terre si sono storicamente avuti tra cultura italiana e slovena. Le prime attestazioni di uso scritto ufficiale dello sloveno ci portano a un'epoca che vede la possibilità di un dialogo fecondo tra le due culture e sono legate alla figura di Primož Trubar (1508-1586), il 'padre della letteratura slovena', che arriva adolescente a Trieste a servizio del vescovo Pietro Bonomo, conosciuto a Vienna, che lo avvicina all'umanesimo latino e lo spinge all'uso pastorale dello sloveno. Di chiare simpatie luterane, Trubar conoscerà Pier Paolo Vergerio il Giovane, già vescovo di Capodistria e scomunicato perché passato alla Riforma, che lo indurrà all'adozione dell'alfabeto latino per la resa dei fonemi dello sloveno e darà alle stampe molti libri in sloveno tra cui un catechismo e una traduzione dei Salmi e del Nuovo Testamento. La traduzione e la diffusione popolare della Bibbia sono un passaggio che nella storia di altre lingue minoritarie d'Italia è del tutto assente. Se la prima traduzione della Bibbia in sloveno è del 1584, la prima traduzione in sardo nuorese, a cura di Salvatore Ruju, è del 2003 e in friulano, a cura di don Francesco Placereani e don Antonio Bellina, del 1984 (p. 115). Al Cinquecento rissale anche il primo vocabolario quadrilingue latinotedesco-sloveno-italiano fatto dal grammatico tedesco $\mathrm{H}$. Megiser che si era accostato allo sloveno nel circolo dei protestanti sloveni di Tubinga. Ma la repressione della Controriforma frena purtroppo bruscamente lo sviluppo delle lettere slovene. Facciamo un salto e arriviamo ai secoli cruciali per il deterioramento nel rapporto tra comunità italiana e slovena a Trieste. La grande stagione della letteratura triestina in sloveno, non diversamente dalla grande stagione della letteratura triestina in italiano, corre parallela al massimo sviluppo nel corso dell'Ottocento del grande emporio marittimo (nato dall'istituzione per volontà imperiale del porto franco nel 1719). L'anno cruciale è il 1848 che insieme al risveglio nazionale slavo vede l'inizio delle tensioni con la comunità italiana, che vive male la neocostituita Associazione slava di Trieste, come poi il movimento politico Edinost (Unità) con il relativo quotidiano, fino alla costruzione del Narodni dom (la Casa della cultura, nota anche come Hotel Balkan) costruito nel 1904 da Max Fabiani. Essa era il simbolo della comunità slovena: il 25\% dei 229.510 abitanti che contava Trieste al censimento austriaco del 1910 si dichiarava sloveno. Più di quanti ne contasse allora la stessa Lubiana: 41.000. Era Trieste, di fatto, la capitale culturale del popolo sloveno (p. 112). La rimozione dell'altro iniziata a metà Ottocento, nel momento in cui l'altro non era più disposto a rinunciare alla propria identità per ottenere l'integrazione nel tessuto borghese cittadino, si aggrava di molto con il passaggio della città al Regno d'Italia: il Narodni dom viene incendiato il 13 luglio 1920 senza alcun intervento a protezione dall'aggressione da parte della forza pubblica. E le cose poterono solo peggiorare con l'avvento al potere di Mussolini: l'italianizzazione forzata (degli stessi nomi!), divieto di uso pubblico dello sloveno, poi la guerra e la dura repressione dei resistenti. Dopo l'8 settembre del 1943 vi fu l'annessione del Litorale al Terzo Reich e l'istitu- 
zione nella Risiera di Trieste (una fabbrica dismessa per la pilatura del riso) dell'unico campo di sterminio dell'Europa occidentale con il suo corredo di tortura e di morte; i famosi 40 giorni in cui la città tra il 1 maggio e il 12 giugno del 1945 fu sotto il controllo di Tito, con le vendette e la giustizia sommaria che gettava altre vittime nelle foibe del Carso che andavano ad aggiungersi a quelle della repressione nazifascista. La pacificazione e la normalizzazione tarda ad arrivare: l'ex provincia del Terzo Reich, e ora progettato Territorio Libero di Trieste, è divisa in Zona A, fino al 1954 sotto il controllo del Governo Militare Alleato che permette nuovamente l'uso dello sloveno ma non ufficiale, e zona B, amministrata dalla Yugoslavia. Fino al 1975 quei confini non sono riconosciuti come confini politici. Nel mezzo la nuova tragedia umana degli esuli. Le ferite come si vede sono molte e profonde, antiche e recenti. Il risultato è che «il dialogo tra la cultura italiana e quella slovena non è ancora aperto" (Ibid.) e lo dimostra la scarsa conoscenza reciproca e il numero ridotto di traduzioni di autori sloveni in italiano e italiani in sloveno. Emblematico il tardo e improvviso successo italiano di Boris Pahor, nato a Trieste nel 1913 e testimone e protagonista involontario delle terribili vicende del secolo scorso. Necropoli, il libro di memorie sulla sua esperienza nei campi di concentramento nazisti, pubblicato in sloveno nel 1967 è stato tradotto in italiano per la prima volta nel 1997, ma nonostante i riconoscimenti prestigiosi ricevuti in Francia e Germania, il successo in Italia è venuto per caso, dopo essere stato ospite della trasmissione televisiva di Fabio Fazio nel 2008, come ricorda egli stesso nell'intervista in fondo al volume (p. 192).

Il saggio offre dunque molte opportunità di riflessione e arricchimento dell'informazione su un argomento così rilevante, fornendo una notevole quantità di dati bibliografici, in nota e in appendice, e un'impressionante mole di indicazioni di autori e opere (a volte purtroppo in modo elencatorio, anche se per comprensibili ragioni di spazio), con una particolare attenzione alla narrativa del secondo dopoguerra. Dispiace però non poco che il volume non sia stato corredato da un indice dei nomi, che l'avrebbe reso uno strumento di studio e di lavoro decisamente più fruibile consentendo di recuperare e collegare più agilmente le informazioni, anche quando variamente dislocate nel corso della trattazione. 